\title{
Tunable magnetic vortex resonance in a potential well
}

\author{
P. Warnicke, ${ }^{1, *}$ P. Wohlhüter,,${ }^{1,2}$ A. K. Suszka, ${ }^{1,2}$ S. E. Stevenson, ${ }^{1}$ L. J. Heyderman, ${ }^{1,2}$ and J. Raabe ${ }^{1}$ \\ ${ }^{1}$ Paul Scherrer Institute, 5232 Villigen PSI, Switzerland \\ ${ }^{2}$ Laboratory for Mesoscopic Systems, Department of Materials, ETH Zurich, 8093 Zurich, Switzerland
}

(Received 5 April 2017; revised manuscript received 23 September 2017; published 3 November 2017)

\begin{abstract}
We use frequency-resolved x-ray microscopy to fully characterize the potential well of a magnetic vortex in a soft ferromagnetic permalloy square. The vortex core is excited with magnetic broadband pulses and simultaneously displaced with a static magnetic field. We observe a frequency increase (blueshift) in the gyrotropic mode of the vortex core with increasing bias field. Supported by micromagnetic simulations, we show that this frequency increase is accompanied by internal deformation of the vortex core. The ability to modify the inner structure of the vortex core provides a mechanism to control the dynamics of magnetic vortices.
\end{abstract}

DOI: 10.1103/PhysRevB.96.174402

\section{INTRODUCTION}

The magnetic vortex [1,2] has generated wide interest due to the rich physics associated with its complex nature and promising functionality in spin-based technology. Although a range of applications has been suggested such as information storage [3], magnetic logic [4,5], and oscillators [6-8], the fundamental properties of the vortex that govern its highfrequency behavior are not fully understood.

In a ferromagnetic square, a well-known low-energydomain configuration is the Landau state, where spins circulate around an out-of-plane vortex core to minimize magnetic stray fields. The magnetostatic energy, which depends on the position of the vortex core, can be summed up into an effective potential, and displacing the core from its equilibrium position creates magnetic charges along the edges of the square and increases the magnetostatic energy. Inside the potential, the lowest energy excitation is the gyrotropic mode $[9,10]$. Experimental attempts to determine the potential of a magnetic vortex using high-frequency excitations have so far led to different conclusions. While the resonance frequency is expected to remain largely unchanged in a harmonic potential [11], observations of both a redshift [12] and a blueshift [13] in the resonance frequency have been attributed to an anharmonicity of the potential well.

Among methods to investigate the dynamic behavior of the vortex, spectroscopic techniques [11,14] have been employed to measure its characteristic frequency. Impedance spectroscopy [11], for example, is usually performed on a large number of structures due to its limited sensitivity and thereby provides an average of the dynamic behavior. Anisotropic magnetoresistance measurements [14] have been performed on a single structure, thus avoiding the problem of averaging over multiple structures with different spin configurations. However, the high current densities required for effective spin transfer induce thermal loads and Oersted fields within the vortex that obscure a straightforward analysis. Magnetic microscopy techniques making use of, for example, the magneto-optic Kerr effect [15] or photoemission [16], provide a direct measure of the spin configuration of the vortex, thus

*peter.warnicke@psi.ch allowing for direct determination of the vortex-core position. However, up to now there has been no successful attempt to quantify the potential well using magnetic imaging.

In this study, we map out the energy potential of the confined vortex using spatially resolved broadband spectroscopy, which allows the resonance frequency and displacement to be determined with the same technique. This extended frequencyresolved mode of scanning transmission X-ray microscopy (STXM) was implemented at the X07DA/PolLux beamline [17] at the Swiss Light Source using X-ray magnetic circular dichroism (XMCD) as a contrast mechanism.

\section{EXPERIMENT}

Thin-film permalloy squares with a side length of $b=6 \mu \mathrm{m}$ were prepared by electron-beam lithography and lift-off processing. Polycrystalline permalloy $\left(\mathrm{Ni}_{80} \mathrm{Fe}_{20}\right)$ with a thickness of $h=50 \mathrm{~nm}$ was sputtered onto $100-\mathrm{nm}$-thick silicon nitride membranes and capped with $1 \mathrm{~nm}$ of Al to prevent rapid oxidation. A stripline consisting of $\mathrm{Ti}(5 \mathrm{~nm}) / \mathrm{Cu}(200 \mathrm{~nm}) / \mathrm{Ti}(5 \mathrm{~nm})$ was subsequently patterned on top of the squares, and magnetic microwave field excitations were generated in the sample with currents transmitted through the stripline. An upper limit of the magnetic field intensity in the sample was estimated from the field $\mu_{0} J / 2 w$ generated by a planar conductor where $w=10 \mu \mathrm{m}$ is the width of the stripline and $J$ is the transmitted current. The transmitted currents are measured at the output of the stripline with a real-time oscilloscope. To allow for XMCD measurements of the in-plane magnetization, the sample normal is tilted $30^{\circ}$ away from the incoming x-ray beam.

Temporal evolution of the magnetization is imaged via time-resolved XMCD in a pump-probe scheme at the $\mathrm{Ni}$ $L_{3}$ absorption edge $(852.7 \mathrm{eV})$. Timing signals from the synchrotron are used to lock an arbitrary function generator with the data acquisition system [see Fig. 1(a)]. Current signals from the generator are amplified and passed through the stripline shown in Fig. 1(a), where the resulting magnetic fields inductively excite the sample. The magnetic field excitation is synchronized with the probing photon pulses from the synchrotron. An acquisition system based on a field-programmable gate array sorts the detector signal into $N$ time channels. Ultimately, the time resolution in this experiment is limited by the width of the photon bunches of 
(a)
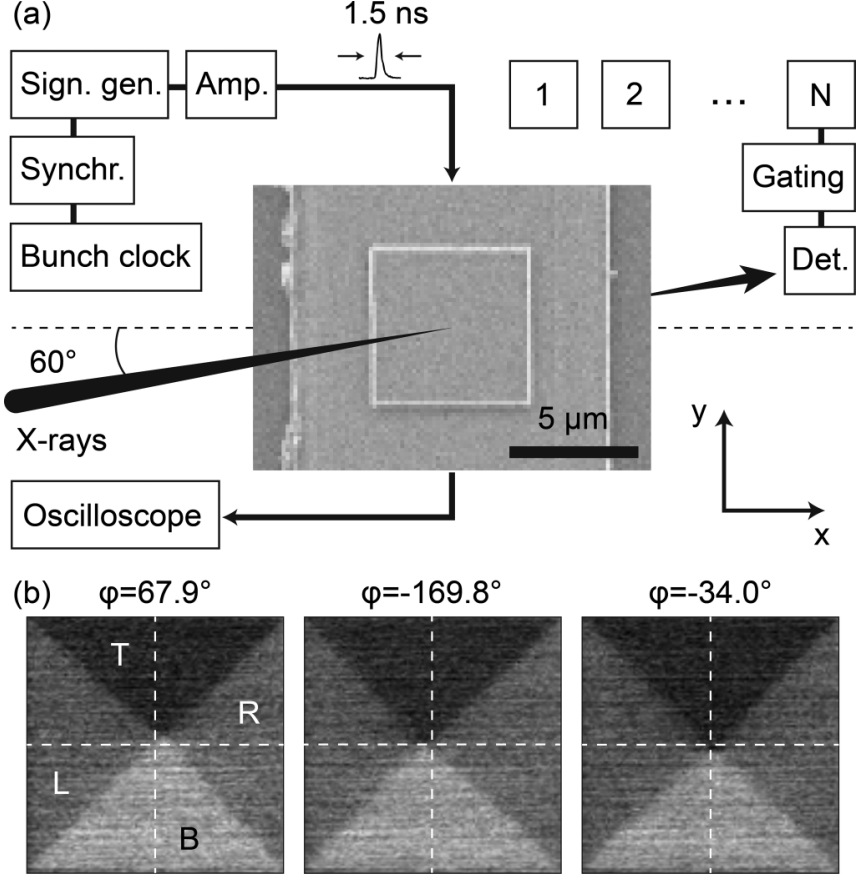

FIG. 1. (a) Schematic diagram of the experimental setup with a scanning electron microscopy image of the permalloy square and stripline. Timing signals from the synchrotron bunch clock are synchronized with an arbitrary waveform generator (sign. gen.) and the data acquisition system. Current signals from the generator (continuous wave or pulses) are amplified and passed through the stripline where the resulting magnetic fields inductively excite the sample. The magnetic field excitation is synchronized with the x-ray bunches. An acquisition system based on a field-programmable gate array sorts the detected x-ray signal into $N$ time channels. (b) Time-resolved XMCD images of the square acquired at various phases $\varphi$ of the continuous wave excitation. The four magnetic domains in the system are marked with $T$ (top), $B$ (bottom), $R$ (right), and $L$ (left).

the synchrotron ( $\sim 100 \mathrm{ps})$. Access to the frequency domain of the magnetization is obtained by broadband excitation and subsequent Fourier analysis of the time-resolved XMCD data.

\section{RESULTS AND DISCUSSION}

\section{A. Depth and width of the potential well}

Before exploring the dynamic properties of the system, we probe the static properties that describe the size of the potential well. Subjected to an applied magnetic field $\mathbf{B}=-B_{x} \hat{\mathbf{e}}_{x}$ the vortex core is displaced along the $y$ axis (coordinate axes indicated in Fig. 1) and finds a new equilibrium at a distance $\delta$ away from the center. The vortex-core position is determined by the intersection of the four magnetic domains $T$ (top), $B$ (bottom), $R$ (right), and $L$ (left) in Fig. 1(b). A gradually increasing magnetic field $B_{x}$ displaces the magnetic vortex core toward the edge of the square until it starts to annihilate at a breakdown field of $B_{0}=4.66 \pm 0.24 \mathrm{mT}$, providing a value for the depth of the potential well. This breakdown field occurs at a displacement of $\delta_{0}=1590 \pm 134 \mathrm{~nm}$ corresponding to the half width of the confining potential.

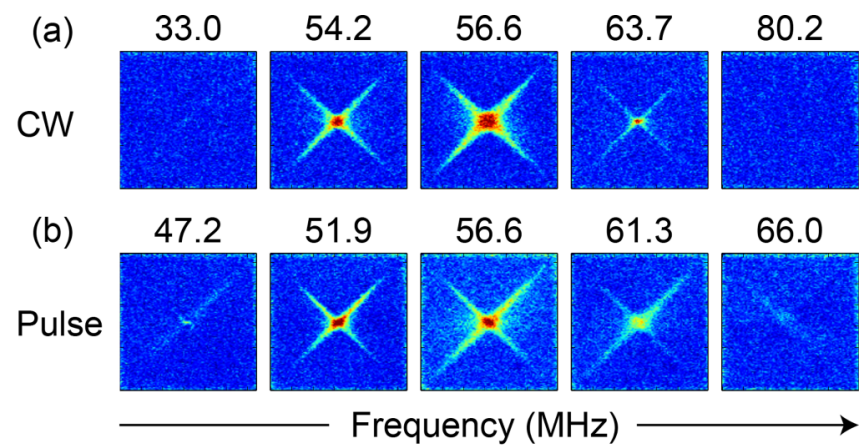

(c)

(d) $\quad 56.6 \mathrm{MHz}$
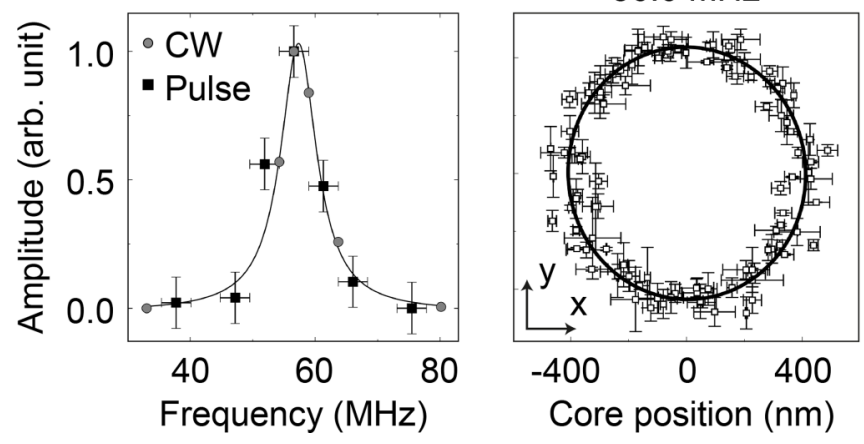

FIG. 2. Fourier amplitude for (a) continuous-wave excitation obtained by time-resolved XMCD imaging at the indicated driving frequencies and (b) pulsed excitation obtained by frequency-resolved XMCD imaging. (c) Normalized Fourier amplitude showing overlapping resonance peaks for the two methods. (d) Vortex core positions for cw excitation at resonance ( $x$ and $y$ scales are the same).

\section{B. Time-resolved characterization of the vortex core dynamics}

Resonant excitations serve as a probe of the dynamic properties. In order to measure the resonance frequency we use two different excitation methods: continuous wave (cw) and pulsed excitation. Starting with $\mathrm{cw}$, the vortex core is excited with a small $\mathrm{cw}$ magnetic field and the dynamic response is imaged. In order to limit the dynamics to the linear regime, we use excitation field amplitudes $\left(\sim 0.2 B_{0}\right)$ significantly below the saturation field [18]. XMCD images are acquired every $2 \mathrm{~ns}$ with respect to the cw excitation yielding a phase-resolution limit of $\Delta \varphi=2 \pi / N$ [19]. XMCD images of the square at various phases of gyration of the vortex core about its equilibrium position are shown in Fig. 1(b). The spatially resolved motion of the vortex can be represented by the amplitude of the Fourier-transformed time-resolved magnetic images $[15,16]$. In Fig. 2(a), the Fourier amplitude is shown for various excitation frequencies. With increasing frequency the orbit of the vortex-core motion increases until it passes a maximum indicating the resonance frequency of the system. While the resonance frequency of the core $(\sim 57 \mathrm{MHz})$ is much lower than that of the domain walls ( $\sim \mathrm{GHz})$, the lower frequency motion of the core still forces the domain walls to oscillate. The composite motion of the core and domain walls is evidenced by the enhanced Fourier amplitude in the central region and along the diagonals. Vortex-core positions during cw excitation at the peak frequency are shown in Fig. 2(d). In contrast to spin structures with large cores, such as bubbles and skyrmions, which display cycloidal motion [20,21], we 
(a)

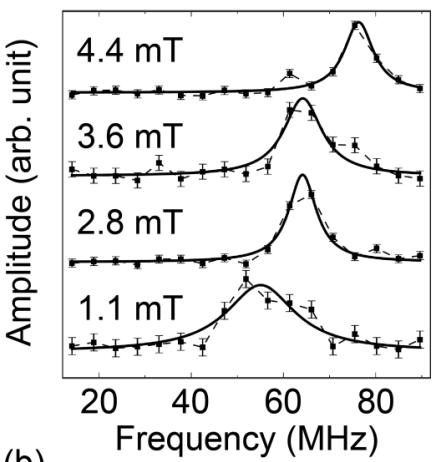

(b)

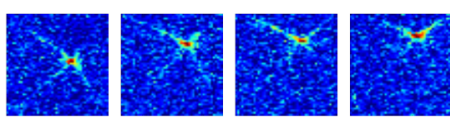

$1.1 \mathrm{mT} 2.8 \mathrm{mT} 3.6 \mathrm{mT} 4.4 \mathrm{mT}$

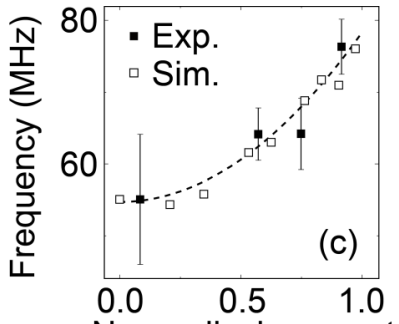

Norm. displacement

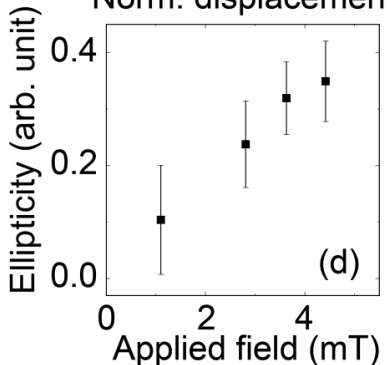

FIG. 3. (a) Frequency spectra showing the normalized Fourier amplitude for pulsed excitation in the presence of magnetic fields along the $x$ axis. Vertical offsets between the spectra have been added for visual clarity. (b) Spatially resolved Fourier amplitude at the resonance frequency for each applied field. (c) Evolution of the resonance frequency as a function of normalized vortexcore displacement $\delta / \delta_{0}$. The experimental data are compared with micromagnetic simulations, where the frequencies obtained from micromagnetic simulations have been normalized to the experimental zero-field frequency by a scaling factor of 0.72 . The dashed line is a guide to the eye indicating the increasing frequency trend. (d) Ellipticity $\eta=1-w_{y} / w_{x}$, where $w_{x}$ and $w_{y}$ are the widths of the Fourier amplitude peak at resonance along $x$ and $y$, respectively.

observe a nearly circular path of the vortex core during gyration as indicated by the elliptical fit (solid line).

\section{Frequency-resolved characterization of the vortex core dynamics}

Instead of sweeping the cw excitation frequency in multiple measurement scans, the resonance frequency can be determined in a single scan using a fast magnetic-field pulse to excite multiple modes in the system. A Fourier transformation of the time-resolved data provides the response at all available frequencies, which facilitates the determination of the resonance frequency in an unknown system. The Fourier amplitude for pulsed excitation in Fig. 2(b) displays a maximum response near the expected resonance frequency. Comparing the two methods [Fig. 2(c)], we find that the measured resonance peaks coincide. Fitting a Lorentzian to the $\mathrm{cw}$ data yields a peak resonance at $57 \mathrm{MHz}$ and a linewidth of $7 \mathrm{MHz}$. The fact that the resonance peaks determined by the two methods match well demonstrates that frequency-resolved STXM is a useful tool to characterize the dynamic response of an excited vortex core.

In order to determine the frequency response of the vortex at different core positions inside the potential well, we excite the core with a pulse in combination with static magnetic bias fields $-B_{x} \hat{\mathbf{e}}_{x}$. We observe an increasing frequency trend (blueshift) with increasing $B_{x}$ as shown in Fig. 3(a). At the spectral frequency peak, a small region of high intensity is present in the spatially resolved Fourier amplitude, as

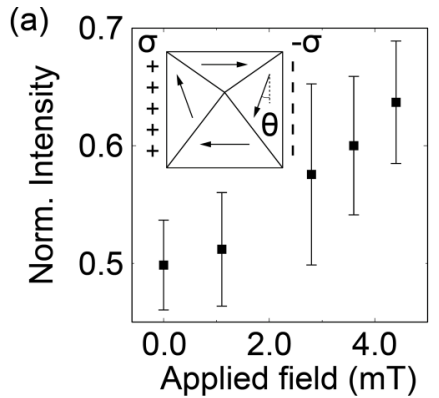

(c)

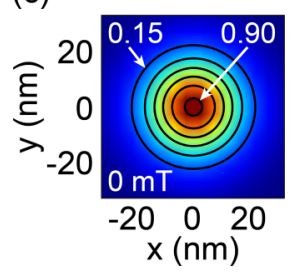

(d)

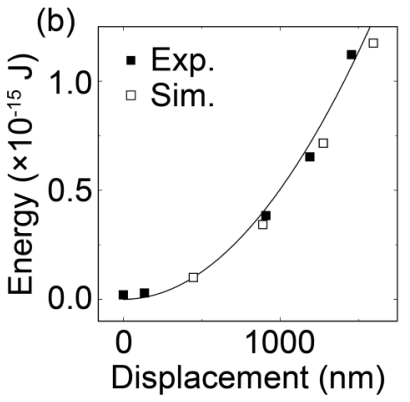

(e)
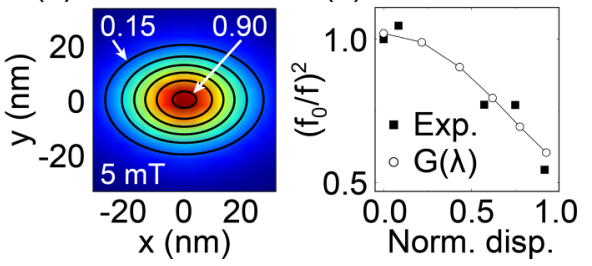

FIG. 4. (a) Normalized XMCD intensity $\left(I_{R}-I_{B}\right) /\left(I_{T}-I_{B}\right)$. The inset illustrates the spin configuration in an applied magnetic field. Spins in the $L$ and $R$ domains tilt toward the field direction with an angle $\theta$. (b) Magnetostatic energy $U$ of the magnetic vortex calculated from Eq. (1) with a parabolic fit to the experimental data (solid line). Simulated out-of-plane magnetization component of the vortex core at (c) zero field and (d) at $B_{x}=5 \mathrm{mT}$. (e) The experimentally measured $\left(f_{0} / f\right)^{2}$ marked with solid squares, where $f$ is the resonance frequency and $f_{0}$ is the resonance frequency at zero field, shows qualitative agreement with the expression $G(\lambda)=$ $\left(\lambda^{2}+\lambda\right) / 2+0.02$, marked by the connected hollow points, where $\lambda=\lambda_{y} / \lambda_{x}$ is the ratio of the simulated vortex core widths along $y$ and $x$. The applied magnetic fields for the experimental data points are $0,1.1,2.8,3.6$, and $4.4 \mathrm{mT}$ and increase with increasing normalized vortex-core displacement $\delta / \delta_{0}$.

shown in Fig. 3(b) for each applied magnetic field, as a result of increased vortex-core gyration. With increasing applied magnetic field, the vortex-core displacement increases gradually [Figs. 3(b) and 3(c)] in accordance with the evolution of the peak position in the Fourier amplitude map in Fig. 3(b). We note that the blueshift is accompanied by a compression of the gyrotropic motion along the displacement direction. To obtain an estimate of the compression, we determine the ellipticity of the gyrotropic motion $\eta=1-w_{y} / w_{x}$ from the widths $w_{x}$ and $w_{y}$ of the peak along $x$ and $y$, respectively, in the Fourier amplitude map. This ellipticity amounts to $0.104,0.238,0.319$, and 0.349 for $1.1,2.8,3.6$, and $4.4 \mathrm{mT}$, respectively, and is shown in Fig. 3(d).

\section{Mapping the potential well}

From here we proceed to determine the size and shape of the potential well. The magnetostatic energy $U=$ $\mu_{0} \int H_{d} M_{s} d V / 2$ can be obtained by integration over the sample volume, which in our case can be divided into four subvolumes corresponding to the $T$ (top), $B$ (bottom), $L$ (left), and $R$ (right) domains of the system. The demagnetization field $H_{\mathrm{d}}$ can be estimated as $\mathbf{H}_{d}=N_{x} \sigma \hat{\mathbf{e}}_{x}$ using the demagnetization factor $N_{x}[22,23]$ and the magnetic charge $\sigma$ which builds up on the side of the square as a result of spin misalignment [see inset of Fig. 4(a)]. While the domain with spins aligned 
parallel to the field $(B)$ grows at the expense of the antiparallel domain $(T)$, its spins are not subjected to the Zeeman torque and therefore maintain their alignment. Consequently, there are no surface charges in these domains. In contrast, the combined size of the $L$ and $R$ domains stays approximately constant and their spins tilt with an angle $\theta$ in the direction of the applied magnetic field due to the Zeeman torque. With $\sigma=\mathbf{M} \cdot \mathbf{n}=M_{s} \sin \theta$ we can now express the potential as

$$
U=\left(\frac{1}{2} b \sin \theta+\delta\right) \frac{\mu_{0}}{2} N_{x} M_{s}{ }^{2} b h \sin \theta,
$$

where the first and second terms inside the parentheses come from integration over $L+R$ and $B+T$ domains, respectively, and $h(b)$ is the thickness (side length) of the square. XMCD is a contrast mechanism that measures the projection of spins along the X-ray propagation vector. By measuring the XMCD intensities one can obtain the spin-tilt angle in the $L$ and $R$ domains from $\sin \theta=2\left(I_{R}-I_{B}\right) /\left(I_{T}-I_{B}\right)-1$, where $I_{R}, I_{B}$, and $I_{T}$ are the XMCD intensities of the $R, B$, and $T$ domains, respectively. The experimentally determined potential is presented in Fig. 4(b) and can be fitted with $U=\kappa y^{2} / 2$ as shown by the solid line, indicating a potential with harmonic shape. In the analysis above we have not accounted for the domain-wall contribution to the potential energy. The magnitude of this energy $U_{\mathrm{dw}}$ can be estimated by first noting that the total length of the domain walls increases with increasing vortex core displacement. Assuming negligible bending of the domain walls, an upper limit of the energy can be expressed as $U_{\mathrm{dw}}=$ $0.092 b h \gamma$ where $\gamma$ is the energy density of the domain wall.

\section{E. Micromagnetic simulations}

To elucidate the behavior of the vortex core we carried out micromagnetic simulations [24] using the MUMAX code [25]. A constant aspect ratio $h / b$ was used in the simulations so that the resonance frequency has the same order of magnitude [10] as in the experimental system. Simulations were carried out by applying gradually increasing magnetic field amplitudes in steps of $\sim 0.1 B_{0}$ and recording the relaxation of the magnetization to equilibrium after each step. In contrast to anharmonic systems, which display a dependence of the frequency on the pulse amplitude, we found that varying the pulse amplitudes from $0.1 B_{0}$ to $0.5 B_{0}$ results in essentially the same frequency (within $\sim 1 \%$ ), suggesting that the system behaves as a harmonic oscillator in this amplitude range. The normalized frequencies obtained from simulations at various applied magnetic fields are plotted in Fig. 3(c) and match well the experimental frequencies, which are taken from Fig. 3(a).

Evolution of the internal spin configuration of the vortex core in an applied magnetic field was determined by performing micromagnetic simulations on a square magnetic element with the same dimensions as the experimental geometry. It can be seen from the out-of-plane magnetization component in the core region at zero magnetic field [Fig. 4(c)] that the core has equal widths $\lambda_{x}$ and $\lambda_{y}$ along $x$ and $y$, respectively. As the field is increased, the core is gradually compressed along $y$ (and stretched along $x$ ) until it reaches a point where $\lambda_{y} / \lambda_{x}=0.69$ at $5 \mathrm{mT}$ [Fig. 4(d)]. This compression can be understood by considering the two opposing forces acting on the vortex core: the restoring force $\mathbf{F}=-d U / d y \hat{\mathbf{e}}_{y}$ pulls the core toward the bottom of the potential well, while the opposing force -F from the applied field torque stabilizes the core. The core deformation is linked to a change in the resonance frequency $f$ of the vortex [see Fig. 4(e)]. We find that the experimentally observed $\left(f_{0} / f\right)^{2}$ is in qualitative agreement with $\left(\lambda^{2}+\lambda\right) / 2+0.02$, where $f_{0}$ is the resonance frequency at zero field and $\lambda=\lambda_{y} / \lambda_{x}$, indicating an increasing resonance frequency with increasing vortex-core deformation.

Spins on the edge of the structure may also contribute to the frequency increase. An increase in the out-of-plane magnetization component at the edge, $M_{z \text {,edge }}$, is expected to lead to a change in the gyrocoupling coefficient [9], which is proportional to the difference between the out-of-plane components in the center of the core and at the edge of the structure. For a standard vortex this difference is equal to the polarity $p$. In the case of $M_{z \text {,edge }}>0$, e.g., due to displacement $\delta$ of the vortex core toward the edge, the gyrocoupling coefficient will be proportional to the $p-M_{z \text {,edge }}$ and, since the $f$ is inversely proportional to the gyrocoupling

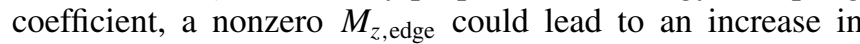
$f$. We have analyzed the effect from the edge region using micromagnetic simulations. However, the average $M_{z \text {,edge }} / M_{\mathrm{s}}$ shows no significant increase with increasing $\delta$ and remains below $5 \times 10^{-6}$, which is comparable to the standard deviation of $M_{z \text {,edge }}$. While we do not see any increase in $M_{z \text {,edge with }}$ increasing $\delta$, we speculate that such an effect might be more pronounced in small geometries, where the region occupied by the core is larger relative to the structure.

A rigid vortex model based on the Thiele equation can be used to estimate the resonance frequency for a displaced vortex core in a disk in the thin-film limit (aspect ratio $\ll 1)$ [26]. For the same aspect ratio as our square $(h / b=0.008)$, the model predicts a frequency increase $\Delta f$ of about $8 \%$ at the maximum experimental displacement. This is an underestimation compared to both the experimental observation $(\Delta f=39 \%)$ and the micromagnetic simulations $(\Delta f=38 \%)$. However, since the model assumes that the vortex core remains rigid, any change in the resonant behavior due to a deformation of the core is not captured.

\section{F. Vortex core mass}

It could be envisioned that the highly localized structure of the magnetic vortex leads to a description of a particle that can exhibit particle-like properties, such as mass. In a simple picture, the concept of mass can be seen as the resistance to acceleration which suggests that mass and dynamic behavior are mutually dependent. For a vortex, the mass is commonly understood to be zero, although the topic of vortex mass is not free from debate. Theoretical work, based on the helical deformation of a moving vortex in an infinite medium, has shown that the translational mode of the vortex is noninertial and exists only in the presence of external forces [27]. However, the situation is different for a vortex in a finite medium where the confinement leads to the appearance of a potential well with a restoring force acting on the vortex, and it has been argued that some form of vortex mass is needed to describe the vortex-core motion in confined geometries [28,29]. By considering a harmonic potential, the experimentally obtained resonance frequencies correspond to a mass $m \propto 1 / f^{2}$ [28], suggesting 
that a frequency blueshift is analogous to a reduction of mass. This would further suggest that the compression of the core [Fig. 4(e)] is linked to a decreasing vortex mass. In this respect, the direct identification of resonance frequency by frequency-resolved magnetic imaging establishes a powerful tool to probe inertial and noninertial modes in order to explore the existence of vortex mass in confined magnetic systems.

\section{CONCLUSIONS}

The experimentally observed blueshift (see Fig. 3) agrees qualitatively with recent observations for a vortex in a cylindrical dot [13], but is in direct contrast to the case of a pinned vortex where a decreasing frequency trend (redshift) was measured with increasing magnetic field [12]. Our study provides an explanation to these contrasting behaviors, demonstrating that a magnetic-field-induced deformation of the vortex core determines the change (positive or negative) in the resonance frequency of the vortex core.

In summary, we employed frequency-resolved x-ray microscopy to determine the link between dynamic behavior and the internal spin structure of a confined magnetic vortex.
A complete characterization of the energy potential is made possible by direct imaging of the spin configuration and its dynamic response. An applied magnetic field leads to a blueshift of the resonance frequency of the system. Micromagnetic simulations show that the frequency shift is accompanied by a deformation of the vortex core. Such deformations provide an explanation for the contrasting behavior of redshift [12] and blueshift [13] observed for magnetic vortices in different geometrical confinements, as well as for the variation in resonance frequency reported for free and pinned vortices [30]. This work demonstrates magnetic-field-induced deformations of the vortex core as an alternative method to control the resonance frequency of a magnetic vortex.

\section{ACKNOWLEDGMENTS}

The authors would like to thank M. Horisberger for support with sample fabrication. The experiments were carried out at the X07DA Pollux beamline at the Swiss Light Source. Funding from the German Minister für Bildung und Forschung (BMBF) through Contracts No. 05KS4WE1/6 and No. 05KS7WE1 is acknowledged. The study was supported by the Swiss National Science Foundation (SNF).
[1] T. Shinjo, T. Okuno, R. Hassdorf, K. Shigeto, and T. Ono, Science 289, 930 (2000).

[2] A. Wachowiak, J. Wiebe, M. Bode, O. Pietzsch, M. Morgenstern, and R. Wiesendanger, Science 298, 577 (2002).

[3] S. K. Kim, K. S. Lee, Y. S. Yu, and Y. Choi, Appl. Phys. Lett. 92, 022509 (2008).

[4] H. Jung, Y.-S. Choi, K.-S. Lee, D.-S. Han, Y.-S. Yu, M.-Y. Im, P. Fischer, and S.-K. Kim, ACS Nano 6,3712 (2012)

[5] D. Kumar, S. Barman, and A. Barman, Sci. Rep. 4, 4108 (2014).

[6] N. Locatelli, A. Hamadeh, F. Abreu Araujo, A. D. Belanovsky, P. N. Skirdkov, R. Lebrun, V. V. Naletov, K. A. Zvezdin, M. Muñoz, J. Grollier, O. Klein, V. Cros, and G. de Loubens, Sci. Rep. 5, 17039 (2015).

[7] V. S. Pribiag, I. N. Krivorotov, G. D. Fuchs, P. M. Braganca, O. Ozatay, J. C. Sankey, D. C. Ralph, and R. A. Buhrman, Nat. Phys. 3, 498 (2007).

[8] A. Dussaux, B. Georges, J. Grollier, V. Cros, A. V. Khvalkovskiy, A. Fukushima, M. Konoto, H. Kubota, K. Yakushiji, S. Yuasa, K. A. Zvezdin, K. Ando, and A. Fert, Nat. Commun. 1, 8 (2010).

[9] D. L. Huber, J. Appl. Phys. 53, 1899 (1982).

[10] K. Yu. Guslienko, B. A. Ivanov, V. Novosad, Y. Otani, H. Shima, and K. Fukamichi, J. Appl. Phys. 91, 8037 (2002).

[11] V. Novosad, F. Y. Fradin, P. E. Roy, K. S. Buchanan, K. Yu. Guslienko, and S. D. Bader, Phys. Rev. B 72, 024455 (2005).

[12] D. Bedau, M. Kläui, M. T. Hua, S. Krzyk, U. Rüdiger, G. Faini, and L. Vila, Phys. Rev. Lett. 101, 256602 (2008).

[13] O. V. Sukhostavets, B. Pigeau, S. Sangiao, G. de Loubens, V. V. Naletov, O. Klein, K. Mitsuzuka, S. Andrieu, F. Montaigne, and K. Y. Guslienko, Phys. Rev. Lett. 111, 247601 (2013).

[14] S. Kasai, Y. Nakatani, K. Kobayashi, H. Kohno, and T. Ono, Phys. Rev. Lett. 97, 107204 (2006).

[15] I. Neudecker, K. Perzlmaier, F. Hoffmann, G. Woltersdorf, M. Buess, D. Weiss, and C. H. Back, Phys. Rev. B 73, 134426 (2006).
[16] J. Raabe, C. Quitmann, C. H. Back, F. Nolting, S. Johnson, and C. Buehler, Phys. Rev. Lett. 94, 217204 (2005).

[17] J. Raabe, G. Tzvetkov, U. Flechsig, M. Böge, A. Jaggi, B. Sarafimov, M. G. C. Vernooij, T. Huthwelker, H. Ade, D. Kicoyne, T. Tyliszczak, R. H. Fink, and C. Quitmann, Rev. Sci. Instrum. 79, 113704 (2008).

[18] K.-S. Lee and S.-K. Kim, Appl. Phys. Lett. 91, 132511 (2007).

[19] We use $N=212$ time channels. A higher $N$ would yield higher phase resolution, but the large loss in photon intensity per channel outweighs this advantage.

[20] I. Makhfudz, B. Krüger, and O. Tchernyshyov, Phys. Rev. Lett. 109, 217201 (2012).

[21] K.-W. Moon, B. S. Chun, W. Kim, Z. Q. Qiu, and C. Hwang, Phys. Rev. B 89, 064413 (2014).

[22] $N_{x}=0.01463$ obtained using the method in Ref. [23].

[23] A. Aharoni, J. Appl. Phys. 83, 3432 (1998).

[24] Simulations were performed on the experimental sample geometry using a cell size of $5 \mathrm{~nm}$ except for the dynamic simulations in Fig. 3(c), which were performed on a scaled geometry $(600 \times 600 \times 5 \mathrm{~nm})$ and a $5 \times 5 \times 2.5-\mathrm{nm}$ cell size. Standard parameters for permalloy were used: saturation magnetization $M_{s}=800 \mathrm{kAm}^{-1}$, exchange constant $A=1.3 \times 10^{-11} \mathrm{Jm}^{-1}$, and Gilbert damping parameter $\alpha=0.02$.

[25] A. Vansteenkiste, J. Leliaert, M. Dvornik, M. Helsen, F. GarciaSanchez, and B. Van Waeyenberg, AIP Adv. 4, 107133 (2014).

[26] Y. Gaididei, V. P. Kravchuk, and D. D. Sheka, Int. J. Quantum Chem. 110, 83 (2010).

[27] A. V. Nikiforov and E. B. Sonin, Zh. Eksp. Teor. Fiz. 85, 642 (1983) [Sov. Phys. JETP 58, 373 (1983)].

[28] G. M. Wysin, Phys. Rev. B 54, 15156 (1996).

[29] K. Y. Guslienko, G. N. Kakazei, J. Ding, X. M. Liu, and A. O. Adeyeye, Sci. Rep. 5, 13881 (2015).

[30] D. Bedau, M. Kläui, S. Krzyk, U. Rüdiger, G. Faini, and L. Vila, Phys. Rev. Lett. 99, 146601 (2007). 